\title{
The Hyperdense Middle Cerebral Artery Sign in Drip-and-Ship Models of Acute Stroke Management
}

\author{
Lise Jodaitis $^{a} \quad$ Noémie Ligot $^{a} \quad$ Rudy Chapusette $^{\mathrm{b}} \quad$ Thomas Bonnet $^{\mathrm{c}}$ \\ Nicolas Gaspard ${ }^{a}$ Gilles Naeije ${ }^{a}$ \\ ${ }^{a}$ Department of Neurology, Erasme Hospital, ULB, Brussels, Belgium; ${ }^{b}$ Department of \\ Radiology, Erasme Hospital, ULB, Brussels, Belgium; ${ }^{C}$ Department of Interventional \\ Neuroradiology, Erasme Hospital, ULB, Brussels, Belgium
}

Keywords

Acute stroke imaging · Hyperdense middle cerebral artery sign · Acute stroke management

\section{Abstract}

Background: Large vessel occlusion (LVO) leads to debilitating stroke and responds modestly to recombinant tissue plasminogen activator (rt-TPA). Early thrombectomy improves functional outcomes in selected patients with proximal occlusion but it is not available in all medical facilities. The best imaging modality for triage in an acute stroke setting in drip-andship models is still the subject of debate. Objectives: We aimed to assess the diagnostic value of millimeter-sliced noncontrast computed tomography (NCCT) hyperdense middle cerebral artery sign (HMCAS) in itself or associated with clinical data for early detection of LVO in drip-and-ship models of acute stroke management. Methods: NCCT of patients admitted to the Erasme Hospital, ULB, Brussels, Belgium, for suspicion of acute ischemic stroke between January 1 and July 31, 2017, were collected. Patients with brain hemorrhages were excluded, leading to 122 cases. The presence of HMCAS on NCCT was determined via visual assessment by 6 raters blinded to all other data. An independent rater assessed the presence of LVO on digital subtraction angiography imaging or contrast-enhanced CT angiography (CTA). The sensitivity, false-positive rate (FPR), and accuracy of HMCAS and the dot sign to detect LVO were calculated. The interobserver agreement of HMCAS was assessed using Gwet's AC1 coefficient. Then, on a separate occasion, the first 2 observers rereviewed all NCCT provided with clinical clues. The sensitivity, FPR, and accuracy of HMCAS were recalculated. Results: HMCAS was found in $21 \%$ of the cases and a dot sign was found in $9 \%$. The mean HMCAS sensitivity was $62 \%$ (95\% Cl 45-79\%) and its accuracy was $86 \%$ (95\% Cl 79-92\%) for detecting 
LVO. The interobserver reliability coefficient was $80 \%$ for HMCAS. Combined with clinical information, HMCAS sensitivity increased to $81 \%(95 \% \mathrm{Cl} 68-94 ; p=0.041)$ and accuracy increased to $91 \%$ (95\% Cl 86-96\%). Conclusion: When clinical data are provided, detection of HMCAS on thinly sliced NCCT could be enough to decide on transfer for thrombectomy in drip-and-ship models of acute stroke management, especially in situations where CTA is less available and referral centers for thrombectomy fewer and further apart.

(C) 2020 The Author(s)

Published by S. Karger AG, Basel

\section{Introduction}

Stroke is the second cause of death and the main cause of acquired disability [1]. Most debilitating strokes are due to proximal arterial occlusion (i.e., internal carotid artery, M1/ M2 segment of the middle cerebral artery, A1 segment of the anterior cerebral artery, and basilar and intracranial vertebral arteries), which accounts for $40 \%$ of all ischemic strokes [2]. Thrombectomy improves functional outcomes in selected patients with large vessel occlusion (LVO) [3-7] but is not available in all medical facilities. To meet that issue, acute stroke management led to intra and extra hospital re-organization. In extra hospital management, the development of "hub-and-spoke" stroke networks, where hospitals able to provide recombinant tissue plasminogen activator (rt-TPA) are organized around a referral center that performs thrombectomy, increased the number of stroke interventions $[8,9]$. However, despite widening of the time window therapy to 16-24 h [10], many patients are still excluded from recanalization therapy due to a lack of viable brain tissue to salvage. One of the most common causes is the time lost in hospital-to-hospital transfer [11].

In this work, we aim to assess the place of a simple and widely available noncontrast computed tomography (NCCT) indirect sign of LVO, i.e., the hyperdense middle cerebral artery sign (HMCAS), to identify earlier a subset of patients with LVO and gain time for treatment in drip-and-ship models of acute stroke management. We thus determined the diagnostic value and the interrater reliability of the HMCAS and the dot sign (which is the equivalent of the HMCAS within the Sylvian fissure) using millimeter-sliced NCCT in isolation or in combination with clinical information for early detection of LVO strokes

\section{Materials and Methods}

\section{Study Design and Sample}

Patients who were admitted to the emergency department of a tertiary academic medical center (Hôpital Erasme) with a suspected acute ischemic stroke and who benefited from an NCCT between January 1 and July 31, 2017, were retrospectively included. Spontaneous intracranial hemorrhage visible on the initial NCCT and patients without proper brain and cervical vessel injection imaging were excluded. The remaining NCCT were then anonymized by removing the patient's name and image markings and they were given a randomly assigned number. Demographic and clinical data such as the National Institutes of Health Stroke Scale (NIHSS) at admission and the final diagnosis were retrieved from the patients' medical records.

\section{Standard of Care}

At our institution, all patients presenting to the emergency department with acute $(<7.5 \mathrm{~h}$ or of uncertain timing) neurologic symptoms benefit from an immediate bedside 
evaluation, including neurological assessment with the NIHSS and NCCT imaging. In the absence of clinical or radiological contraindications, patients admitted within $4.5 \mathrm{~h}$ of onset are given IV rtTPA in the imaging suite; they then undergo further imaging with a contrast-enhanced CT angiography (CTA) to assess extra- and intracranial arteries. When iodine contrast injection is contraindicated due to an iodine intolerance or renal failure, subjects benefit from magnetic resonance angiography (MRA). Patients with LVO identified on CTA who are admitted within $7.5 \mathrm{~h}$ of onset and have a premorbid modified Rankin scale score $<2$ are eligible for thrombectomy. Patients with wake-up strokes or strokes admitted $>4.5 \mathrm{~h}$ after onset receive a perfusion CT before thrombolysis or thrombectomy is performed, based on mismatch criteria.

\section{Imaging Parameters and Review}

The imaging parameters were: continuous axial slice acquisition from the vertex to the skull base, a slice thickness of $1.5 \mathrm{~mm}$, an increment thickness of $1.0 \mathrm{~mm}$, a tube current of $165 \mathrm{mAS}$, a tube voltage of $120 \mathrm{kv}$, and $64 \times 0.6 \mathrm{~mm}$ collimation.

First, in order to determine HMCAS and dot sign reliability, anonymized NCCT were retrospectively presented and independently reviewed by 6 raters blinded to all clinical and other imaging data, i.e., 4 neurologists (N.G., L.J., N.L., and G.N.), 1 neuroradiologist (R.C.), and 1 interventional neuroradiologist (T.B.). The presence or absence of HMCA and dot signs was determined by visual assessment and, respectively, defined as a relative hyperdensity of an MCA in comparison to its contralateral counterpart and as the hyperdensity of an arterial structure (seen as a dot on axial slices) in the Sylvian fissure relative to the contralateral side or to other vessels within the Sylvian fissure [12].

Then, to determine the yield of the HMCAS in a more practical real-life situation, 2 of the initial raters ( 2 clinical neurologists, i.e., L.J. and G.N.) reassessed the presence of the HMCAS and the dot sign after gaining access to the patient's clinical details.

\section{Statistical Analysis}

Positive HMCA and dot signs detected by clinicians were considered true positives if a proximal MCA (M1, M2) occlusion was confirmed on arterial CTA, MRA, or digital subtraction angiography (DSA). Means and 95\% CI for sensitivity, the false-positive rate (FPR), and the accuracy of both signs, and of the presence of any of the two, were calculated. The interrater reliability for the HMCAS and dot sign was assessed using Gwet's AC1 coefficient, a chanceadjusted index for the reliability of categorical measurements that has been shown to provide a more stable interrater reliability coefficient than Cohen's $\kappa$ [13]. Means and 95\% CI for Gwet's AC1 were calculated using leave- $N$-out jackknife resampling with 100 iterations, leaving $50 \%$ of the sample out at each iteration. Then, the sensitivity, the FPR and the accuracy of both signs, and of the presence of any of the 2 , were reassessed by 2 of the initial raters who had access on that occasion to the laterality and extent of the neurological findings on examination. Data are presented as means $( \pm$ SD), means $(95 \% \mathrm{CI})$, or numbers $(\%)$. Differences in test performance with and without clinical information were assessed using log-linear analysis in a model containing 4 variables (sign [present or absent], proximal occlusion [present or absent], rater [1 vs. 2], and clinical information [available vs. not available]). Statistics were performed in R (The R Foundation, Vienna, Austria).

\section{Results}

Patients

The patients demographic and clinical features summarized in Table 1. One hundred forty-nine potential subjects were admitted during the study period. We excluded 27 of them, 


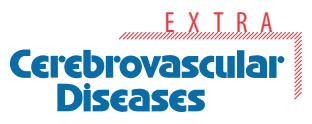

Table 1. Patient demographics and clinical features

\begin{tabular}{l|l}
\hline Cerebrovasc Dis Extra 2020;10:36-43 \\
\hline DOI: 10.1159/000506971 & $\begin{array}{l}\text { ○ 2020 The Author(s). Published by S. Karger AG, Basel } \\
\text { www.karger.com/cee }\end{array}$ \\
\hline
\end{tabular}

Jodaitis et al.: The HMCAS Sign in Drip-and-Ship Models of Acute Stroke Management

\begin{tabular}{lc} 
Age, years & $71 \pm 17$ \\
Sex, male & $70(57)$ \\
Confirmed ischemic stroke & $84(69)$ \\
Stroke mimics & \\
$\quad$ TIA & $12(10)$ \\
$\quad$ Epilepsy & $9(7)$ \\
$\quad$ Psychogenic & $8(6)$ \\
$\quad$ Other etiologies & $9(7)$ \\
Ischemic stroke with proximal occlusion & $34(28)$ \\
$\quad$ IC+M1 & 12 \\
$\quad$ Proximal M1 & 14 \\
$\quad$ Distal M1 or M2 & 8 \\
NIHSS & \\
$\quad$ In patients with confirmed stroke & $10 \pm 7$ \\
In patients with LVO & $15 \pm 6$ \\
\hline
\end{tabular}

The total number of patients is 122 . Data are presented as means \pm SD or numbers (\%). TIA, transient ischemic attack; IC, internal carotid artery; M1, first segment of the MCA; M2, second segment of the MCA (other etiologies included: multiple sclerosis, $n=1$; intoxication, $n=1$; brain tumor, $n=1$; neurologic degradation due to metabolic/infectious causes in patients with preexisting deficits, $n=3$; vestibular neuritis, $n=1$; radial nerve palsy, $n=1$; and migraines, $n=1$ ).

Table 2. Interobserver agreement and accuracy of the HMCAS and the dot sign when blinded to clinical data

\begin{tabular}{llllll}
\hline & Gwet's AC1 & PA & Se & FPR & Mean accuracy \\
\hline HMCAS & $80(73-87)$ & $70(62-78)$ & $62(45-79)$ & $5(1-10)$ & $86(79-92)$ \\
Dot sign & $87(82-91)$ & $75(68-83)$ & $19(6-32)$ & $6(1-10)$ & $73(65-81)$ \\
HMCAS or dot sign & $73(65-80)$ & $63(54-71)$ & $68(51-84)$ & $11(5-17)$ & $83(77-90)$ \\
\hline
\end{tabular}

Data are presented as mean percents (95\% CI). PA, percent agreement; Se, sensitivity.

i.e., 15 due to acute intracerebral hemorrhage and 12 due to a lack of adequate imaging performed at our institution (7 had NCCT in an outside hospital prior to admission to our institution, 1 had a brain MRI without a previous NCCT, and 4 did not get the angiographic sequences [CTA, MRA, or DSA] within the desired time window). Thus, 122 patients were included in this study.

\section{HMCAS on NCCT when Blinded to Clinical Data}

The HMCAS was identified in 21\% (95\% CI 18-26) and the dot sign in 9\% (95\% CI 5-11) of the cases. The interrater reliability for the HMCAS, the dot sign, or the presence of any of the 2 signs was substantial (Gwet's AC1 $=80 \%, 95 \%$ CI 73-87), almost perfect (Gwet's AC1 = 87\%, 95\% CI 82-91), and substantial (Gwet's AC1 = 73\%, 95\% CI 65-87), respectively. The sensitivity and FPR of the HMCAS were 62\% (95\% CI 45-79) and 5\% (95\% CI 1-10), respectively, yielding a very good test accuracy of $86 \%$ (95\% CI 79-92). The dot sign had a much lower sensitivity (19\%, 95\% CI 6-32) and a similar FPR (6\%, 95\% CI 1-10). The presence of 
Table 3. Accuracy of signs without and with a clinical clue

\begin{tabular}{llcc}
\hline & Se & FPR & Accuracy \\
\hline HMCAS reader 1 without clinical information & $62(45-79)$ & $2(0-5)$ & $88(82-93)$ \\
HMCAS reader 2 without clinical information & $62(44-80)$ & $10(4-17)$ & $82(75-89)$ \\
HMCAS reader 1 with clinical information & $77(62-91)$ & $3(0-7)$ & $91(86-96)$ \\
HMCAS reader 2 with clinical information & $85(73-97)$ & $6(1-11)$ & $92(87-97)$ \\
\hline
\end{tabular}

Data are presented as mean percents (95\% CI). Se, sensitivity.

either the HMCAS or the dot sign only marginally improved the sensitivity (68\%, 95\% CI 51-84) compared to the HMCAS but with a higher FPR (11\%, 95\% CI 5-17), resulting in a marginally lower accuracy (83\%, 95\% CI 77-90). See Table 2.

\section{HMCAS on NCCT Combined with Clinical Data}

Disclosure of clinical information to the raters significantly improved the sensitivity and accuracy of the HMCAS ( $p=0.04$ for the effect of clinical information). We found no significant difference between the 2 raters. See Table 3 .

\section{Discussion/Conclusion}

The HMCAS was found in $21 \%$ of cases suspicious for acute stroke, with good interobserver agreement. When clinical data were provided, as in real-life situations, the HMCAS on millimeter-sliced NCCT led to $81 \%$ sensitivity and 91\% accuracy for MCA proximal occlusion detection, which could help to identify earlier a subset of patients with LVO in drip-and-ship models of acute stroke management.

Despite the study's retrospective design and the sample size, the population studied mirrors the baseline characteristics of the patients included in the major trials on thrombectomy for proximal occlusion in terms of age and NIHSS $[3,6]$. Similarly, the proportion of proximal occlusions within our cohort of ischemic strokes (40.5\%) parallels previous prospective reports [2], as does the average NIHSS score of our patients with LVO [14]. Finally, the stroke etiology distribution in our population was comparable to that in a recent study that analyzed the associations between HMCAS and stroke etiology [15]. So, the evidence provided by our study is likely to be transposable to most acute stroke settings.

The HMCAS is typically found in $17-50 \%$ of acute MCA occlusions and has a specificity of $95 \%$, with a lower sensitivity of $52 \%$ according to a recent meta-analysis that reviewed studies from 1990 to 2013 [16]. In our study, its sensitivity was of $62 \%$ for raters blinded to clinical information and $77-82 \%$ when raters were aware of clinical details, which was thus higher than expected. This can be explained by both technical and practical differences. First, previous studies have demonstrated that using thinner $(<3 \mathrm{~mm})$ NCCT slices increases the sensitivity of the HMCAS for detecting LVO, as the average diameter of the intracranial artery diameter is $<3 \mathrm{~mm}$ [17]. In our acute stroke management, we systematically use 1.5 -mm-slice section CT, whereas most prior studies have been conducted with $\geq 5 \mathrm{~mm}$ slices. Second, the raters in our study are clinicians and radiologists routinely involved in acute stroke management, which probably led to the high interrater reliability coefficient of 0.80 and the $81 \%$ sensitivity for LVO when clinical data were added to the model. This is a message but 
also a limitation of our study, because our results may not be generalizable to settings where NNCT are not interpreted by stroke specialists.

The advantage of adding clinical data has already been highlighted by Lim et al. [18] in a study that assessed the sensitivity and specificity of the HMCAS on thin-slice (1.5 mm) NCCT combined with the NIHSS score and a brief clinical history and found up to $79 \%$ sensitivity for LVO in patients with an NIHSS $>10$ [18]. It also parallels results from a recent study that demonstrated that combining the NIHSS score and the absolute attenuation value of the middle cerebral artery on NCCT showed $85 \%$ sensitivity for LVO [19].

The good accuracy of the HMCAS on NCCT for LVO when assessed by stroke clinicians is an important element in the global context of acute stroke management choice of settings. Indeed, the American Stroke Association recommends: "transport patients rapidly to the closest available appropriate institution to provide initial emergency care, including administration of intravenous rt-TPA and if needed, to arrange transfer in centers capable of performing endovascular stroke treatment with comprehensive per-procedural care" [20]. This drip-and-ship model effectively increases the rate of stroke interventions [8] but is also associated with longer transfer times that may delay endovascular treatments [11, 21]. Several approaches have been tried to prevent interhospital delays between primary and secondary stroke centers in suspected LVO, i.e., by-passing time-consuming imaging in patients arriving soon after symptom onset (like perfusion CT or MRI that can induce almost a 20-min delay in acute ischemic stroke treatment [22]), relying on telemedicine, angiography suites, and mobile strokes. In that context, our findings support that HMCAS on thin slices NCCT could efficiently identify 8 out of 10 patients with acute MCA LVO and allow earlier transfers for thrombectomy in a substantial subset of patients. However, these findings do not argue for the replacement of CTA by NCCT in drip-and-ship models of acute stroke management as $20 \%$ of LVO would be missed. Indeed, CTA provides almost $100 \%$ accuracy for LVO [23] and important information on stroke etiology and should remain the first imaging for triage in acute stroke management. However, it is not realized around the clock in many primary stroke centers and may not be feasible for technical problems or contraindications. Furthermore, the yield and prognosis value of CTA are higher when CTA is realized close to the thrombectomy procedure, even in the angiography suite [24-26].

So, shipping suspected LVO based on identification of the HMCAS on thin-slice NCCT in order to perform CTA in the thrombectomy stroke center may both be efficient and adequate in selected situations. Finally, many middle-income countries that now account for a large part of stroke mortality and related disability [27] are likely to develop stroke networks based on the drip-and-ship model for economic reasons. So, the presence of HMCAS on 1.5-mm NCCT could be enough to take the "drip-and-shift" decision in LVO and gain precious time for endovascular treatment.

Another limitation of this study is the focus on MCA occlusions that prevents our results from being applied to other sites of proximal occlusion. However, carotid and MCA thrombi represent the vast majority of LVO (respectively, 24\% within the carotid artery and 52\% within the proximal MCA) while ACA and posterior circulation occlusions occur only in a minority of cases (1 and 16\%, respectively) [28]. However, NCCT arterial hyperdensity signs in studies that looked at both anterior and posterior circulation LVO yielded sensitivity results similar to those for MCA occlusions [18]. So, it is likely that our findings may still be valid for proximal posterior occlusions.

In conclusion, in drip-and-ship models of acute stroke management, detection of the HMCAS on millimeter-sliced NCCT could identify earlier a subset of patients with LVO and hurry the transfer for thrombectomy in primary stroke centers, especially in situations where CTA is less available and referral centers are fewer and further apart. However, even combined with clinical data, the HMCAS on NCCT still misses $20 \%$ of LVO, which warrants the use of CTA when feasible. 


\section{Acknowledgement}

Pasquale Scoppettuolo helped to collect data.

\section{Statement of Ethics}

The study protocol was approved by the research institute's committee on human research. The authors have no ethical conflicts to disclose.

\section{Disclosure Statement}

The authors have no conflict of interests to declare.

\section{Funding Sources}

None.

\section{Author Contributions}

Lise Jodaitis: main writer. Gilles Naeije: substantial contributions to the conception and design of this work, acquisition of data, critical revision of this work, and final approval of the version for publication. Noémie Ligot, Rudy Chapusette, and Thomas Bonnet: substantial contributions to acquisition of data, critical revision of this work, and final approval. Nicolas Gaspard: substantial contributions to acquisition, analysis, and interpretation of data for this work, critical revision of this work, final approval.

\section{References}

1 Murray CJ, Lopez AD. Mortality by cause for eight regions of the world: Global Burden of Disease Study [Internet]. Lancet. 1997 May;349(9061):1269-76. [cited 2018 Aug 11].

2 Heldner MR, Zubler C, Mattle HP, Schroth G, Weck A, Mono ML, et al. National Institutes of Health stroke scale score and vessel occlusion in 2152 patients with acute ischemic stroke. Stroke. 2013 Apr;44(4):1153-7.

3 Campbell BC, Mitchell PJ, Kleinig TJ, Dewey HM, Churilov L, Yassi N, et al.; EXTEND-IA Investigators. Endovascular therapy for ischemic stroke with perfusion-imaging selection [Internet]. N Engl J Med. 2015 Mar; 372(11):1009-18.

4 Jovin TG, Chamorro A, Cobo E, de Miquel MA, Molina CA, Rovira A, et al.; REVASCAT Trial Investigators. Thrombectomy within 8 hours after symptom onset in ischemic stroke [Internet]. N Engl J Med. 2015 Jun;372(24): 2296-306.

5 Saver JL, Goyal M, Bonafe A, Diener HC, Levy EI, Pereira VM, et al.; SWIFT PRIME Investigators. Stent-retriever thrombectomy after intravenous t-PA vs. t-PA alone in stroke [Internet]. N Engl J Med. 2015 Jun;372(24): 2285-95. [cited 2018 Aug 11].

6 Goyal M, Demchuk AM, Menon BK, Eesa M, Rempel JL, Thornton J, et al.; ESCAPE Trial Investigators. Randomized assessment of rapid endovascular treatment of ischemic stroke [Internet]. N Engl J Med. 2015 Mar;372(11): 1019-30. [cited 2018 Aug 11].

7 Berkhemer OA, Fransen PS, Beumer D, van den Berg LA, Lingsma HF, Yoo AJ, et al.; MR CLEAN Investigators. A randomized trial of intraarterial treatment for acute ischemic stroke [Internet]. N Engl J Med. 2015 Jan; 372(1):11-20.

8 Rymer MM, Thurtchley D, Summers D. Expanded modes of tissue plasminogen activator delivery in a comprehensive stroke center increases regional acute stroke interventions [Internet]. 2003. Available from: https:// doi.org/10.1161/01.STR.0000071107.66925.93. 
9 Price CI, Clement F, Gray J, Donaldson C, Ford GA. Systematic review of stroke thrombolysis service configuration [Internet]. 2009. Available from: https://doi.org/10.1586/14737175.9.2.211.

10 Nogueira RG, Jadhav AP, Haussen DC, Bonafe A, Budzik RF, Bhuva P, et al. Thrombectomy 6 to 24 hours after stroke with a mismatch between deficit and infarct. N Engl J Med. 2018 Jan 4;378(1):11-21.

11 Prabhakaran S, Ward E, John S, Lopes DK, Chen M, Temes RE, et al. Transfer delay is a major factor limiting the use of intra-arterial treatment in acute ischemic stroke. Stroke. 2011 Jun;42(6):1626-30.

12 Barber PA, Demchuk AM, Hudon ME, Pexman JH, Hill MD, Buchan AM. Hyperdense sylvian fissure MCA "dot" sign: A CT marker of acute ischemia. Stroke. $2001 \mathrm{Jan}$;22(1):84-8.

13 Wongpakaran N, Wongpakaran T, Wedding D, Gwet KL. A comparison of Cohen's Kappa and Gwet's AC1 when calculating inter-rater reliability coefficients: a study conducted with personality disorder samples [Internet]. BMC Med Res Methodol. 2013 Apr;13(1):61.

14 Maas MB, Furie KL, Lev MH, Ay H, Singhal AB, Greer DM, et al. National Institutes of Health Stroke Scale score is poorly predictive of proximal occlusion in acute cerebral ischemia [Internet]. Stroke. 2009 Sep;40(9): 2988-93. [cited 2018 Aug 11].

15 Kim SK, Baek BH, Lee YY, Yoon W. Clinical implications of CT hyperdense artery sign in patients with acute middle cerebral artery occlusion in the era of modern mechanical thrombectomy. J Neurol. 2017 Dec;264(12): 2450-6.

16 Mair G, Boyd EV, Chappell FM, von Kummer R, Lindley RI, Sandercock P, et al.; IST-3 Collaborative Group. Sensitivity and specificity of the hyperdense artery sign for arterial obstruction in acute ischemic stroke. Stroke. 2015 Jan;46(1):102-7.

17 Kim EY, Lee SK, Kim DJ, Suh SH, Kim J, Heo JH, et al. Detection of thrombus in acute ischemic stroke: value of thin-section noncontrast-computed tomography [Internet]. Stroke. 2005 Dec;36(12):2745-7. [cited 2018 Aug 14].

18 Lim J, Magarik JA, Froehler MT. The CT-Defined Hyperdense Arterial Sign as a Marker for Acute Intracerebral Large Vessel Occlusion. J Neuroimaging. 2018 Mar;28(2):212-6.

19 Martins-Filho RK, Dias FA, Alves FFA, Camilo MR, Barreira CMA, Libardi MC, et al. Large vessel occlusion score: a screening tool to detect large vessel occlusion in the acute stroke setting. J Stroke Cerebrovasc Dis. 2019 Apr; 869-75.

20 Powers WJ, Derdeyn CP, Biller J, Coffey CS, Hoh BL, Jauch EC, et al. 2015 American Heart Association/American Stroke Association Focused Update of the 2013 Guidelines for the Early Management of Patients With Acute Ischemic Stroke Regarding Endovascular Treatment. Stroke. 2015;3020-35.

21 Bonafe A, Diener H, Levy E, Baxter B, et al. Analysis of workflow and time to treatment and the effects on outcome in endovascular treatment of acute ischemic stroke: results from the SWIFT. Radiology. 2016;279(3); 888-97.

22 Meretoja A, Strbian D, Mustanoja S, Tatlisumak T, Lindsberg PJ, Kaste M. Reducing in-hospital delay to 20 minutes in stroke thrombolysis. Neurology. 2012 Jul;79(4):306-13.

23 Tan IY, Demchuk AM, Hopyan J, Zhang L, Gladstone D, Wong K, et al. CT angiography clot burden score and collateral score: correlation with clinical and radiologic outcomes in acute middle cerebral artery infarct. AJNR Am J Neuroradiol. 2009 Mar;30(3):525-31.

24 Albers GW, Lansberg MG, Kemp S, Tsai JP, Lavori P, Christensen S, et al. A multicenter randomized controlled trial of endovascular therapy following imaging evaluation for ischemic stroke (DEFUSE 3). Int J Stroke. 2017 Oct;12(8):896-905.

25 Lansberg MG, Lee J, Christensen S, Straka M, De Silva DA, Mlynash M, et al. RAPID automated patient selection for reperfusion therapy: A pooled analysis of the echoplanar imaging thrombolytic evaluation trial (EPITHET) and the diffusion and perfusion imaging evaluation for understanding stroke evolution (DEFUSE) study. Stroke. 2011 Jun;42(6):1608-14.

26 Yamamoto N, Yamamoto Y, Yamaguchi I, Ishihara M, Miyamoto T, Korai M, et al. Cone beam-computed tomography angiography by intravenous contrast injection is reliable to evaluate patients with large vessel occlusion. J Clin Neurosci. 2019 Dec;70:67-71.

27 Kalkonde YV, Alladi S, Kaul S, Hachinski V. Stroke prevention strategies in the developing world. Stroke. 2018 Dec;49(12):3092-7.

28 Galimanis A, Jung S, Mono M-L, Fischer U, Findling O, Weck A, et al. Endovascular therapy of 623 patients with anterior circulation. Stroke. 2012 Apr;43(4):1052-7. 